
internationales

vol. $27-n^{\circ} 1 \mid 2011$

La construction de l'altérité dans l'espace noir atlantique : Etats-Unis - France - Caraïbes - Amérique latine

\title{
Les processus d'ethnicisation et de raci(ali)sation dans la France contemporaine : Africains, Ultramarins et « Noirs »
}

Processes of Ethnicization and Raci(ali)zation in the Contemporary French

Context: Africans, Caribbeans and "Blacks"

Los procesos de etnicización y racialización en la Francia contemporánea:

Africanos, Caribeños y «Negros»

\section{Christian Poiret}

\section{(2) OpenEdition}

Édition électronique

URL : https://journals.openedition.org/remi/5365

DOI : 10.4000/remi.5365

ISSN : $1777-5418$

Éditeur

Université de Poitiers

\section{Édition imprimée}

Date de publication : 1 juin 2011

Pagination : 107-127

ISBN : 979-10-90426-00-9

ISSN : 0765-0752

Référence électronique

Christian Poiret, « Les processus d'ethnicisation et de raci(ali)sation dans la France contemporaine Africains, Ultramarins et « Noirs »», Revue européenne des migrations internationales [En ligne], vol. 27 $n^{\circ} 1$ | 2011, mis en ligne le 01 juin 2014, consulté le 14 avril 2022. URL : http://journals.openedition.org/ remi/5365; DOI : https://doi.org/10.4000/remi.5365 


\section{Les processus d'ethnicisation et de raci(ali)sation dans la France contemporaine : Africains, Ultramarins et « Noirs »}

\section{Christian POIRET ${ }^{1}$}

T es biologistes ont beau avoir démontré qu'il n'existe pas de « races $^{2}$ »

Lhumaines pouvant être distinguées les unes des autres, l'idée de « race » n’en demeure pas moins socialement efficiente. Les « races » humaines n'existent donc pas et pourtant il existe bien quelque chose ayant à voir avec elles. Ce qui existe, ce sont des groupes racisés, constitués d'individus bien réels qui tendent à être traités comme l'incarnation de leur groupe d'appartenance et qui doivent donc apprendre à faire avec. Pour autant, ces groupes constituent-ils un objet scientifique ? Faut-il faire une sociologie, une anthropologie ou une histoire des « Noirs »? Ou même des relations entre " Noirs » et " Blancs », dans une perspective comparable à celle des Race Relations ? Après et avec bien d'autres (Fanon, 1954 ; Banton, 1977 ; Miles, 1987, par exemple) je répondrai par la négative et j'y substituerai la construction d'un objet processuel et contextualisé. La première partie de cet article sera donc consacrée à préciser les modalités théoriques de construction de cet objet avant de le replacer dans le contexte français contemporain. Un contexte, marqué par l'émergence, ou plutôt la réapparition, de la catégorie " Noirs » dans l'espace public depuis quelques années. La deuxième partie présentera une approche compréhensive du processus subjectif de racisation par lequel on devient « Noir », basée

1 Maître de conférences HDR en sociologie, Université Paris Diderot, Sorbonne Paris Cité, Unité de Recherche Migrations et Société (URMIS, Universités Paris 7 - Nice, IRD, CNRS) ; poiret@ univ-paris-diderot.fr

2 J'utilise le mot « race »- de même que les catégories de désignation racisées comme " Noirs », « Blancs », etc. - entre guillemets dans mes commentaires et analyses pour signifier à la fois l'existence et l'efficience de ces catégories dans le cours des interactions sociales, tout en marquant ma distance d'avec un quelconque fondement biologique ou d'une essence objective pouvant définir en soi les groupes et les individus ainsi désignés. Dans les transcriptions d'entretiens, je n'en mets pas, ne sachant pas si les interviewées auraient souhaité ou non les utiliser, moi-même ne recourant à ce type de catégorie que sous une forme réitérative revenant à m'en servir dans le même sens qu'elles. 
sur l'analyse de la parole de personnes pouvant être ainsi catégorisées. Ce processus d'apprentissage sera découpé en quatre grandes formes, liées mais analytiquement distinctes, qui feront l'objet chacune d'un développement : la racialisation, l'altérisation, la conscientisation et l'adaptation à la situation subordonnée. Enfin, la troisième partie conclusive reviendra sur la dimension collective des processus d'ethnicisation et de racisation et leurs conséquences en termes d'action collective.

\section{CONSTRUIRE UN OBJET DE RECHERCHE PROCESSUEL ET CONTEXTUALISÉ}

La dimension processuelle de mon objet de recherche relève d'une perspective dans laquelle il ne s'agit plus de se demander comment sont traités ou maltraités et comment agissent les individus appartenant à tels ou tels groupes minoritaires ${ }^{3}$, supposés préexistants, mais comment les minoritaires sont construits et produits en tant que minoritaires en étant traités comme tels et comment ils s'adaptent et parfois résistent à cette définition de la situation. Les termes « race », « racial », " ethnie », « ethnique », de même que les termes utilisés pour désigner l'une ou l'autre de leurs spécifications (« Noirs », « Arabes », « africanité », etc.) doivent alors être considérés comme désignant des objets d'étude et non pas comme des catégories d'analyse. Parce que ces objets n'ont pas d'existence hors des rapports sociaux qui les produisent, les reproduisent et les transforment, les concepts que j'utilise pour les analyser désignent des processus (racisation, ethnicisation, essentialisation, naturalisation, réification) et leurs effets à un moment donné (raciste, c'est-à-dire à la fois racisant et racisé ; ethniste, à la fois ethnicisant et ethnicisé, etc.). Ces processus expriment un rapport social de domination-subordination qui fonctionne par référence aux origines, qu'elles soient traitées plutôt sur un mode naturalisant - racisation ou plutôt culturalisant - ethnicisation - ou, le plus souvent, selon une combinaison des deux.

Il est maintenant d'usage en France, de qualifier ce rapport social d'interethnique, pour bien marquer que la production d'ethnicité se fait de chaque côté de la relation, même si le groupe dominant tend généralement à rester dans l'indétermination et à penser ses caractéristiques culturelles comme la culture (légitime, de référence) et non comme de l'ethnicité. Mais sans doute serait-il plus clair de nommer ce type de rapport social « rapport d'origination », un rapport pouvant prendre des formes diverses selon les lieux, les époques et les circonstances : ethnisme, racisme mais aussi ethnocentrisme, xénophobie, nationalisme, etc.

Il est possible de considérer que ces diverses manifestations s'inscrivent dans un continuum qui irait de l'ethnocentrisme, comme trait anthropologique universel nécessaire à la pérennité des groupes humains, jusqu'au racisme institutionnalisé organisant systématiquement certaines formations sociales selon des principes raciologiques, à l'image de

3 Les termes «majoritaire » et « minoritaire » sont ici utilisés dans leur acception sociologique et non pas démographique, c'est-à-dire comme des situations produites dans des rapports de domination-subordination indépendamment de l'importance quantitative des groupes concernés : le majoritaire est celui qui «majore » sa position (son statut, son pouvoir, etc.) en minorant celle d'alter (Guillaumin, 2002 [1972]). 
l'Afrique du Sud de l'apartheid par exemple. En ce sens, toutes relèvent d'un seul champ d'analyse. Cependant, inscrire ces différentes formes d'expression d'un même type de rapport social dans un continuum, ne signifie pas qu'elles ne se distinguent pas par des modalités de construction de l'altérité qualitativement différenciées. Non qu'il s'agisse d'une graduation dans la violence qui les accompagne - toutes peuvent aller jusqu'à l'extermination d'alter - mais dans le degré de durcissement des frontières qui séparent les Nous et les Eux, et d'incommensurabilité entre alter et ego. Selon que la référence à l'origine - réelle ou supposée, imputée ou revendiquée - est construite plutôt sur le mode de l'héritage ou plutôt sur le mode de l'hérédité, il en résulte des définitions de la situation, des représentations du social, des orientations de l'action et des modes de légitimation différenciées. C'est en particulier le cas de l'action publique dont je fais ici l'hypothèse qu'elle varie selon les modalités de construction des groupes et des individus minorisés qui en sont la cible.

Cet objet de recherche est aussi contextuel, parce que l'analyse des processus de construction et de production des minorités ethnicisées et racisées nécessite de les replacer dans leur contexte - historique, social, politique, économique - ne serait-ce qu'afin de permettre des comparaisons dans l'espace et le temps et de repérer des variations et des constantes. Comme les autres rapports sociaux de pouvoir, le rapport d'origination doit être analysé dans ses dimensions idéelles et matérielles et à différentes échelles, du macro au microsocial. D'où l'intérêt de la distinction initiée par Véronique De Rudder (1987), entre rapports sociaux et relations sociales interethniques. Les rapports sociaux « renvoient à la distribution des positions respectives des groupes sur les plans économiques, sociaux, institutionnels, statutaires - distribution qui compose un ordre social tributaire de l'histoire, à l'échelle internationale ou nationale. Les rapports sociaux interethniques sont structurants en ce qu'ils s'arriment historiquement à la division du travail, aux relations internationales, aux échanges inégaux et, donc, aux rapports entre les peuples ou entre groupes sociaux au sein des formations étatico-nationales ». Appréhendés à ce niveau, « ils déterminent des places et des chances et imposent des communautés d'expérience telles que, par elles-mêmes, elles tendent à produire des solidarités et des rivalités définies comme "ethniques" ou "raciales" » (De Rudder, Poiret et Vourc'h, 2000 : 38).

Cependant, à une échelle méso ou micro-sociale plus soumise aux conjonctures et aux configurations locales, l'échelle des relations interethniques ou des relations d'origination, on ne peut présupposer ni de la forme de l'actualisation de ces rapports sociaux ni de leur intensité dans le cours de l'action sociale. "Les relations interethniques s'inscrivent, nécessairement, dans l'ordre structurant des rapports interethniques. Mais, tout en les accomplissant concrètement, elles ne font pas que les refléter. Selon les circonstances, elles peuvent même en entraver la reproduction et, surtout, en infléchir l'actualisation (l'importance relative, les effets d'identification, le déplacement ou le recoupement des frontières) » (De Rudder et alii, $2000: 39$ ).

Il y a donc un double niveau de contextualisation à prendre en compte dans l'analyse des processus de production de populations racisées : le contexte macrosocial dans lequel il s'inscrit - ici, la situation nationale française contemporaine inscrite dans des relations transnationales - et les contextes micro ou mésociaux dans lesquels s'inscrivent les processus d'incorporation individuelle de l'idée de « race ». 


\section{« Noirs », le retour ${ }^{4}$}

En France, jusqu'à la fin de la Deuxième Guerre mondiale, la catégorie « Noirs » ou « Nègres » était d'usage courant tandis que le terme "Africains » servait plutôt à désigner les populations européennes et indigènes d'Afrique du Nord. Ce n'est donc que dans les années 1950-1960 que vont émerger la catégorie «Africains » - au sens actuel d'originaires d'Afrique Subsaharienne - ainsi que celles utilisées pour désigner les originaires d'Outre-Mer. Cette émergence se fera à la faveur de la conjonction de trois facteurs : la censure des expressions trop explicitement racisantes après la chute du nazisme ; le besoin de distinguer les migrants originaires d'Afrique Noire des Ultramarins, en raison de l'enjeu que représentaient les droits liés à la nationalité française suite à la départementalisation des cinq vieilles colonies et aux indépendances africaines ; la prise en compte d'une certaine complexification de la catégorie raciale, sous l'impact des mobilisations collectives des migrants africains d'un côté et ultramarins de l'autre.

Cependant, ces catégories ethnicisantes, se référant à de grandes « aires culturelles », restaient contiguës de la catégorie « Noirs » comme le montraient des entretiens menés au début des années 1990, avec des acteurs institutionnels (Poiret, 1996). Il en ressortait que, d'une part ces catégories, comme n'importe quelle autre catégorie sociale, pouvaient être investies du sens de la race dans un jeu d'euphémisation (Guillaumin, 1994) et que, d'autre part, certains interlocuteurs qui distinguaient les migrants en deux groupes distincts, originaires d'Afrique et d'Outre-Mer, avaient tendance à amalgamer leurs descendants dans la catégorie « Noirs ». Mais ce qui se disait pendant les entretiens restait globalement illégitime dans les discours publics.

La situation a nettement changé depuis quelques années, avec un point de basculement qui peut être situé en 2005, lorsque d'une part, au mois de mars, la catégorie « Noirs » est reprise par des membres de l'élite intellectuelle regroupés autour d'Alain Finkielkraut, qui dénoncent son caractère criminogène en lançant un appel contre « les ratonades anti-blancs $»^{5}$; et d'autre part, lorsqu'en novembre, des intellectuels minoritaires ou sympathisants, revendiquent cette catégorie pour en faire un outil de lutte politique avec la création du CRAN (Conseil Représentatif des Associations Noires de France).

La nouveauté ne tient donc pas tant aux populations dont il est question qu'à la manière de les désigner publiquement. Pour autant, la racisation ne se substitue pas à l'ethnicisation. Celle-ci se manifeste régulièrement à travers la stigmatisation spécifique des Africains autour du thème de la polygamie ou de celle des Antillais à propos de la matrifocalité interprétée comme une absence paternelle, par exemple. Il en résulte d'ailleurs qu'une part des discriminations subies par des personnes susceptibles d'être, par ailleurs, catégorisées comme « noires », peuvent être vécues d'abord en tant qu'Africains ou qu'Antillais plutôt qu'en tant que « Noirs ».

4 Pour une analyse plus développée du contexte de (ré)émergence de la catégorie « Noirs » dans la France contemporaine, voir Poiret (2010b).

5 Avec Elie Chouraqui, Chahdortt Djavann, Jacques Julliard, Bernard Kouchner et Pierre-André Taguieff. 
Il n'en demeure pas moins qu'on peut constater un net changement depuis quelques années quant au recours ouvert au registre de la " race », à tel point que le président de la République lui-même se présente comme : "un homme de sang mêlé à la

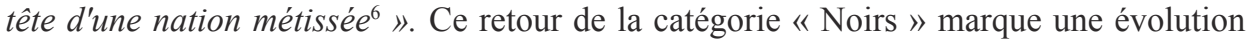
dans le jeu de montré-caché du registre de l'ethnicité et de la " race » caractéristique du modèle assimilationniste français. Le passage de l'ethnicisation à la racisation ne peut pas être tenu pour une simple question sémantique. Il soulève l'hypothèse d'une évolution des rapports interethniques en France, dans le sens d'une tendance générale à l'assignation des populations catégorisées comme «noires » à une situation sociale durablement infériorisée et marginale. Mais ce changement de registre est aussi une forme de reconnaissance de cette tendance, qui exprime la transformation du regard que la société française porte sur elle-même.

\section{UNE APPROCHE COMPRÉHENSIVE DE LA RACISATION}

C'est dans le contexte du tournant de 2005 que j'ai mené une série d'entretiens ${ }^{7}$ sur lesquels je vais m'appuyer pour présenter quelques réflexions sur la dimension subjective des processus par lesquels on devient « Noire », c'est-à-dire femme et « noire » à la fois, dans la France métropolitaine d'aujourd'hui.

Les catégories « raciales » et leurs attributs n'existent pas « en soi » et c'est d'ailleurs pourquoi les mêmes individus peuvent être classés différemment dans le temps ou dans l'espace. Ainsi, un « Métis » vu comme un « Mulâtre » aux Antilles pourra-t-il être catégorisé comme un « Noir » sur le territoire européen de la France. Les catégories « raciales » apparaissent toujours en référence à des jeux d'oppositions catégorielles et à des rapports de domination-subordination souvent doublés de rapports d'exploitation. C'est dans le cours du rapport social raciste qu'est créée la « race ». Elle ne lui préexiste pas. En ce sens, on peut donc dire que toute catégorie « raciale » est aussi une catégorie racisée et racisante et donc une catégorie raciste au sens où elle participe de la reproduction du rapport social raciste (De Rudder et alii, 2000). Dans ce cadre, les processus d'identification ne sont donc pas libres et surtout, ils ne sont ni symétriques, ni équivalents. D'un côté le majoritaire (celui qui majore sa position) se pense comme le référent universel, celui qui ne diffère de rien et par rapport auquel s'ordonne la hiérarchie des Autres ; de l'autre côté, le minoritaire (celui dont la position est minorée) est généralement contraint de construire son identité en contre, en opposition et bien souvent en tentant de retourner le stigmate qui lui est assigné par le majoritaire. À un niveau individuel, les entretiens de recherche sur le vécu du racisme quotidien et des discriminations menés

6 Propos rapportés par le ministre Éric Besson dans l'émission Mots croisés, le 26 octobre 2009.

7 Corpus de quatorze entretiens approfondis (d'une durée variant entre trois heures et huit heures trente), menés en région parisienne avec des jeunes femmes (âgées de vingt-quatre à trente-huit ans), nées ou arrivées très jeunes en France métropolitaine, originaires d'Afrique ou d'OutreMer (s'auto-définissant spontanément comme « Noire » au cours des interviews), diplômées de l'enseignement supérieur (minimum bac +4 ) et, pour la plupart, étant ou ayant été durablement en situation de recherche d'emploi avant ou pendant notre rencontre. Pour plus de détail sur la constitution de ce corpus voir Poiret (2010a). 
avec des Françaises d'ascendances africaines et/ou caraïbéennes, éclairent les conditions d'adoption, volens nolens, d'une identité racisée.

En France, la parole des minoritaires reste globalement peu entendue et peu étudiée. Ceci est probablement lié à la manière dont la question des discriminations racistes a été abordée, non pas sous l'impulsion de mobilisations à la base, mais du haut vers le bas, en particulier sous la pression de l'Union européenne (Guiraudon, 2004). Il en a résulté une prédominance des approches juridiques. Ces approches sont loin d'être inutiles mais elles ont tendance à enfermer les acteurs dans une logique du procès (Wacquant, 1997), qui se retrouve dans la manière dont les plaintes sont traitées. Dans les permanences des associations antiracistes comme à la Halde (Haute Autorité de Lutte contre les Discriminations et pour l'Égalité), la question qui se pose est de savoir quels sont les cas juridiquement traitables. Les autres, au bout du compte, n'ont que peu d'intérêt ou, plus précisément, ils ne servent à rien.

C'est donc dans une tout autre perspective que je me suis appuyé sur l'analyse d'un corpus d'entretiens biographiques, longs et très peu directifs, menés avec des jeunes femmes susceptibles d'être classées comme "Noires ». Ici, le récit biographique ne cherche pas à restituer une cohérence aux histoires de vie. Il est utilisé pour chercher et analyser des situations problématiques par rapport aux normes sociales dominantes en vigueur, en fonction des contextes. C'est ce que Philomena Essed (1995 [1991]) appelle des «scenarii du racisme », comprenant à la fois des attentes quant à ce qui devrait se passer si les personnes étaient traitées « normalement» et à la fois des scripts de traitement racisé potentiels. Ces scénarii peuvent être analysés comme des formes récurrentes d'expression du racisme, ainsi que l'a montré Essed, mais ils peuvent aussi être traités comme des opérateurs de racisation, c'est-à-dire comme des processus par lesquels la « race » est socialement créée et recréée.

Nous avons beau voir spontanément des femmes « noires » en marchant dans la rue, les entretiens montrent que devenir, puis être une « Noire» n'a rien de spontané. C'est ce qu'exprimait clairement une de mes interlocutrices :

\section{Moi, petite, je ne me pensais pas comme Noire. Je me pensais comme enfant, comme collégienne, comme lycéenne. À la fac, j'ai commencé à me penser comme Noire. Et en sortant de la fac, là ce n'était que Noire. Et plus je faisais des démarches [pour trouver du travail] et plus c'était ma hantise. Je voyais Noire. Quand je recevais du courrier, je voyais Noire.}

Devenir « Noire », au moins dans certaines circonstances, relève donc d'un processus qu'il s'agit d'étudier. Pour cela, je développerai une démarche sociologique compréhensive du racisme, basée sur la parole minoritaire. Elle permet d'approcher ce que signifie au quotidien, très concrètement, être confrontée à un ordre social raciste. Car le racisme est aussi une expérience dont on peut faire la sociologie, en interrogeant ce que font les acteurs confrontés à des processus discriminatoires et ségrégatifs. Sur la base d'un traitement inductif de ce corpus, du type théorisation ancrée (Miles et Huberman, 1991), il est possible de dégager quatre grandes formes, liées mais analytiquement distinctes, parmi les processus subjectifs de racisation par lesquels on devient « Noire » : ce sont la racialisation, l'altérisation, la conscientisation et l'adaptation à la situation subordonnée. 


\section{La racialisation}

J'utiliserai le terme racialisation pour désigner un processus cognitif de mise en forme du monde et de définition de la situation, un processus de construction de la réalité sociale, c'est-à-dire la face mentale du racisme compris comme un rapport social. La racialisation reflète les dynamiques de déploiement de l'idéologie raciste que Colette Guillaumin (1992) a analysées selon trois niveaux d'actualisation distincts et hiérarchisés, au sens où chacun nécessite le précédent : le niveau de la perception populaire de sens commun des phénomènes sociaux, une perception plus ou moins non consciente (c'est le discours du " c'est comme ça, il y a des Noirs et des Blancs ») ; le niveau des argumentaires de type prétendument scientifique à visée explicative (c'est le discours du "les "Noirs" ne sont pas comme nous parce que ») ; et le niveau de la doctrine politique qui vise à orienter l'action de l'État (c'est le discours du «donc » et de l'institutionnalisation des catégories racialisantes).

Par distinction, le terme de racisation désigne alors les pratiques et les attitudes orientées et justifiées par la racialisation - consciemment ou non - et qui ont pour effet d'actualiser l'idée de race en produisant des individus et des groupes racisés. C'est un processus de production sociale, la face matérielle du rapport social raciste, faite de discrimination, ségrégation, harcèlement, agression, extermination, etc.

L'intérêt de cette distinction tient à ce que la relation entre racialisation et racisation n'est pas mécanique. D'une part, parce que, même si à une échelle macrosociale la racialisation se traduit par de la racisation, à une échelle interindividuelle, il n'y a pas de lien automatique entre les préjugés et les pratiques (La Piere, 1934). D’autre part, parce qu'un des paradoxes de la situation actuelle tient au fait qu'une partie des politiques publiques et des formes de mobilisation collective qui affichent l'objectif de lutter contre ou de limiter les pratiques qui sont au cœur des processus de racisation aboutissent à renforcer les phénomènes de racialisation. La confusion de ces deux dynamiques comporte donc un risque d'appauvrissement de l'analyse des modes de production des groupes racisés.

Dans le contexte français, sont susceptibles d'être désignées comme « Noirs» les personnes qui présentent les signes d'une ascendance - même lointaine, partielle voire imaginaire - située en Afrique subsaharienne, tandis que la catégorie «Blancs » peut être appliquée à celles qui n'exhibent pas de marqueurs pouvant être interprétés comme manifestant une origine extra-européenne. La différence de modalités de construction de ces deux catégories, l'une « en creux » et l'autre « en plein », est révélatrice de la dichotomie entre un groupe majoritaire qui n'a, le plus souvent, pas besoin de se définir et des groupes minoritaires racisés, les « non-Blancs », définis de manière limitative (Guillaumin, 2002 [1971]).

En fait, le terme « Noirs » est loin d'être la simple désignation d'une caractéristique physique. D'ailleurs personne n'est réellement « noir » ou «blanc » tandis qu'est ouvert tout l'éventail des nuances de pigmentation. La catégorie « Noirs » est une création historique, progressivement construite à l'époque moderne comme un outil légitimant la domination et l'exploitation de certains peuples à travers la traite, l'esclavage, la colo- 
nisation puis les migrations. Et, qu'on le veuille ou non, recourir à la catégorie « Noir » revient à se référer, implicitement ou explicitement, consciemment ou non consciemment, à cette longue histoire de violences, de domination et d'exploitation. Cette forme d'altérisation renvoie donc à des schèmes de pensée raciologiques dont l'intériorisation n'a rien de spontané.

Les entretiens montrent que la racialisation débute par l'intériorisation progressive du fait que certains traits phénotypiques, à commencer par la couleur de peau, sont considérés comme remarquables et qu'ils sont remarqués. Ce pré-requis à l'apprentissage de la signification sociale de la couleur se traduit par la découverte des différences de pigmentation, souvent à l'école maternelle, où on se découvre marron, chocolat, caramel, etc. Puis on comprend que ces caractéristiques sont amalgamées dans une catégorie sociale préexistante, la catégorie « Noire », et surtout que cette catégorie « Noire » s'applique à soi-même.

Donc ma fille, [qui était à l'époque en CE1] rentre un soir et me dit : " oui maman, moi je ne comprends pas, on ne peut pas changer de couleur de peau?" Blessée, je lui demande, mais qu'est-ce qui se passe? Elle fait «non, non, mais j'en ai marre, on me dit que je suis noire... » Je lui dis, ben oui, c'est la couleur de ta peau, tu es noire. Elle me dit «non, je ne suis pas noire, je suis marron, les gens ne comprennent pas que je ne suis pas noire, je suis marron et en plus marron clair.

Donc il faut qu'ils arrêtent de m'appeler la Noire, la sale Noire».

Donc, premier constat, on ne naît pas « Noire », on le devient et on le devient d'abord dans le regard des autres et tout spécialement dans le regard des majoritaires. C'est dans le regard du majoritaire qu'est créée la « différence », une différence syncrétique, amalgame de traits physiques, psychiques, culturels et sociaux. Car apprendre à être « Noire » c'est aussi apprendre que l'on va être jugée sur des apparences, des traits phénotypiques, comme si ces apparences étaient significatives d'une essence particulière sous-jacente. C'est apprendre à être considérée, a priori, non comme un individu mais comme l'incarnation du groupe « Noirs » ou, plus exactement, comme l'incarnation de la représentation que le groupe majoritaire se fait de ce groupe. Apprendre à être « Noire », c'est aussi apprendre - notamment à travers des insultes, des moqueries et des pratiques d'évitement - à connaître les stéréotypes qui expriment « le répertoire limitatif des caractéristiques du groupe » (Guillaumin, 2002 [1972]) et c'est surtout apprendre qu'ils s'appliquent à soi-même.

De ses origines esclavagistes et colonialistes durant lesquelles elle a été utilisée pour forger un groupe infrahumain pouvant légitimement être asservi, la catégorie « Noirs » a hérité d'un registre stéréotypique essentiellement fondé sur l'idée d'une proximité particulière avec la nature et comportant une certaine dose de bestialité pouvant s'exprimer sous une forme positive ("ils ont le rythme dans la peau... ») ou négative (« une infériorité intellectuelle ») mais toujours limitative (Cohen, 1981 ; Dorlin, 2006). Ces représentations proprement naturalisantes sont entremêlées à d'autres qui relèvent des registres social, culturel et psychologique. Bien évidemment la transmission et la légitimation de ces stéréotypes dépassent le cadre des interactions de face-à-face que j'analyse ici, puisqu'elles résultent en grande partie d'actions institutionnelles : reportages médiatiques sur la misère et la violence en Afrique ou en Haïti ; déclarations politiques, des propos de 
Jean-Marie Le Pen sur la supériorité des Noirs sur les Blancs en matière de course à pied ${ }^{8}$ au discours sur « l'homme africain » prononcé à Dakar par Nicolas Sarkozy9 ; clichés publicitaires jouant sur des références au cannibalisme ou sur la beauté sauvage des corps « noirs » (Dewitte, 1990) ; manuels scolaires et animations pédagogiques « interculturelles » véhiculant des clichés misérabilistes pour les uns et folkloriques pour les autres. Pourtant, c'est bien dans le cours d'interactions quotidiennes que les personnes racisées découvrent qu'elles aussi peuvent être tenues pour comptables de ces clichés qui se contredisent parfois entre eux ; que ces stigmates peuvent toujours servir à interpréter et à juger leurs actes ou leurs comportements et qu'il faut donc en tenir compte, même si c'est pour transgresser la norme des comportements attendus (West et Fenstermaker, 1996).

Le maniement de ces stéréotypes permet en particulier de blâmer la victime et d'enseigner à celles qui sont vues comme « Noires » qu'il est toujours possible de leur faire porter la responsabilité des situations défavorables qu'elles rencontrent, par exemple en sous-entendant une absence de motivation ou de débrouillardise comme le racontait une interviewée :

Même l'été, quand on cherche des petits boulots, on ne trouve pas de petits boulots ou on ne trouve pas les mêmes [que les autres étudiants], ils sont plus difficiles, on ne prend pas en compte vos diplômes. Et puis on te dit, " avec tous les diplômes que tu as, tu ne pourrais pas faire autre chose? " Ben oui, c'est tout ce que j'ai trouvé hein?

\section{Un processus global d'altérisation}

La racialisation s'inscrit dans un processus plus global d'altérisation - et c'est ma deuxième grande forme. En deçà de l'intériorisation des stéréotypes qui caractérisent la catégorie « Noire », les entretiens font ressortir un bruit de fond, plus ou moins permanent, composé de manifestations répétitives signalant la « différence » bien que le contenu de celle-ci ne soit pas spécifié.

Ce travail de sape s'exprime en grande partie par du langage non verbal comme ces jeux de regards qu'une des interviewées qualifiait d' "arrêts sur image "; des regards appuyés qui donnent le sentiment d'être passées au crible ou, au contraire, des regards sélectifs au travers desquels les « Noires » passent comme si elles étaient invisibles.

Elle s'exprime aussi à travers un ensemble de formes langagières, comme le tutoiement d'office, et surtout à travers des petites phrases qui manifestent une différence, sans pour autant lui conférer un contenu stéréotypique. Parmi celles-ci, la question des origines, sur le thème " d'où viens-tu ? ", revient comme une rengaine d'autant plus énervante qu'elle est récurrente :

8 Le Monde du 05 avril 2007.

9 « Le drame de l'Afrique, c'est que l'homme africain n'est pas assez entré dans l'histoire. Le paysan africain, qui depuis des millénaires, vit avec les saisons, dont l'idéal de vie est d'être en harmonie avec la nature, ne connaît que l'éternel recommencement du temps rythmé par la répétition sans fin des mêmes gestes et des mêmes paroles ». Discours du 26 juillet 2007. 
On est dans l'ascenseur avec la voisine, il fait chaud en été, on n'a rien à se dire: "Ah il fait chaud, hein? " Ouais, ouais. "Mais vous, vous avez l'habitude dans votre pays. » Mais oui, mais oui. Et bien non, je n'ai pas l'habitude parce que je suis née à Paris, comme vous.

Ces paroles, ces actes, ces comportements constituent bel et bien un apprentissage de l'altérité au terme duquel mes interlocutrices ont appris qu'elles sont vues comme différentes et que, même si cela leur déplaît, elles doivent en tenir compte. Elles font le constat d'une assignation à la différence qui rejoint le sentiment de double conscience que W.E.B DuBois (2007 [1903]) résumait comme le sentiment bizarre d'être à la fois «noir » et américain. Une des conséquences de cette altérisation est que ces jeunes femmes peuvent finir par se sentir étrangères dans leur propre pays. Pour plusieurs d'entre elles, c'est l'expérience d'une discrimination répétitive sur le marché du travail qui les amène à penser qu'elles sont marquées du sceau de l'extranéité. Ainsi, Amina, après avoir multiplié les candidatures pendant ses trois années de chômage, ne reçoit même plus de refus et, quand elle en parle, finit par ne plus trop savoir si elle est française ou non :

Je n'ai même pas de réponse, c'est ça que je ne tolère pas, ce manque de courtoisie. C'est parce que je suis étrangère... bon française... C'est un truc bizarre tout ça, mais donc je suis noire et donc, non, ça ne passe pas.

Cet apprentissage de l'imputation d'une extranéité qui nie leur citoyenneté, renvoie à des enjeux de statuts dans la mesure où la nationalité à laquelle elle est attachée représente une marque statutaire assurant non seulement des avantages, mais aussi des privilèges qui sont refusés aux minoritaires (De Rudder, 1991). Ce processus continu et souvent discret de contestation de leur pleine citoyenneté prépare les "Noires " à des confrontations ultérieures, en leur enseignant qu'elles sont et restent étrangères quoiqu'en dise leur état civil. Plus encore, l'aboutissement de ce travail d'altérisation est l'intériorisation de l'univers symbolique dominant et de l'idéologie raciste qui en fait partie.

Par diffraction du racisme, cette intériorisation peut notamment se traduire par des jeux de distinction entre minoritaires, qui visent à produire des hiérarchisations favorables à ceux qui y ont recours. La ligne de clivage peut alors passer entre « Noirs » et «Arabes », par exemple, mais elle peut aussi traverser le groupe « Noirs », comme lorsque la référence à la traite atlantique et à l'esclavage est utilisée pour opposer «Antillaises » et «Africaines », ainsi que l'évoquaient plusieurs interviewées.

Apprendre à être « Noire » ce n'est pas seulement apprendre que l'on est vue comme différente, comme étrangère, comme « noire », comme potentiellement comptable des stéréotypes attachés à cette catégorie et, à ce titre, stigmatisable. C'est aussi intérioriser que la norme est « blanche » et qu'il est des endroits, des fonctions et des rôles où les « Noires », en général, ne sont pas attendues. C'est ce que constate Diane en revisitant, lors de notre entretien, une de ses expériences de baby-sitting :

Je n'ai pas le souvenir d'avoir gardé des enfants d'une autre origine ou d'une autre couleur, sauf une fois, il y a très longtemps. J'avais parlé avec la mère [au téléphone]. Donc j'avais rendez-vous avec elle et, quand j'ai ouvert la porte, j'ai halluciné parce que la nana, elle était métisse comme moi. Et j'ai vu qu'on s'est 
matées toutes les deux, genre... arrêt sur image renvoyé l'une à l'autre! [rires] Parce qu'en fait, l'une et l'autre, on s'était représenté l'autre comme blanche. Parce que c'est incroyable, même nous, de façon inconsciente, quand on parle à quelqu'un on se dit automatiquement qu'il est blanc, même si nous on est noire ou métisse ou autre. Et moi, je parlais dans ma tête à une Blanche. Et elle dans sa tête, elle parlait à une Blanche aussi. (...) Mais c'est vrai que ça m'avait vraiment interrogée : moi-même je pense que la nana elle est blanche!

Le récit de ce choc, tel qu'il est analysé par Diane, n'est pas sans évoquer la notion de conscience dédoublée [bifurcated consciousness] utilisée par certaines Black feminist (Smith, 1974 ; Hill Collins, 1990) pour qualifier comme un avantage épistémologique la façon qu'ont les personnes minorisées, d'expérimenter le monde social. Une capacité à envisager les choses à la fois dans la perspective du dominant (qui est aussi la perspective dominante) et à la fois dans celle du dominé.

Par leur socialisation, les acteurs ont appris à voir le monde du point de vue des dominants. L'ordre social leur semble évident. Ils s'attendent à trouver certaines personnes dans certaines positions et d'autres non. Dans le cours de la routine quotidienne, ceci « va de soi » et les rapports de domination ne sont pas spontanément perçus en tant que tels. Pourtant, la confrontation, dans une même personne, entre ce point de vue et celui minoritaire rend parfois possible un travail de comparaison et de déconstruction de la fiction d'un ordre social naturel. Il alimente alors un phénomène de conscientisation.

\section{La conscientisation}

Le processus de subjectivation par lequel mes interlocutrices apprennent à devenir « Noire » comprend aussi une dynamique de type politique que j'appelle la conscientisation et qui constitue ma troisième grande forme. Elle s'exprime par une démarche procédant par analyse et par comparaison de cas particuliers à des connaissances plus générales, afin de pouvoir qualifier, ou non, comme racistes certaines situations.

Lorsqu'elles expliquent le déroulement d'une interaction par le fait qu'elles ont été traitées en tant que "Noire », elles le font de manière argumentée en se référant à ce qui est usuellement considéré comme la norme, en fonction des circonstances. Cette capacité d'expertise se fonde sur l'expérience, directement vécue ou transmise par leur entourage, les médias ou la littérature (Essed, 1995 [1991]).

Ces expériences fondatrices relèvent le plus souvent de confrontations directes et répétitives aux discriminations dans le monde du travail et de l'emploi, alors même que les interviewées se sont beaucoup investies dans des formations professionnalisantes en croyant aux valeurs méritocratiques de la République. C'est dans le contraste entre les promesses d'aveuglement à la couleur de l'idéologie républicaine et le vécu de la réalité des discriminations racistes que se forge ce savoir politique, cet esprit critique au sens le plus positif du terme.

L'impact du rapport social raciste sur le fonctionnement d'institutions supposées être organisées sur la base de la seule logique économique ou bureaucratique, donne à voir 
un aperçu de ce que signifie un ordre social racisé : un ordre où les discriminations fonctionnent comme une des modalités de régulation des possibilités différenciées d'accès aux ressources accordées aux un-e-s et aux autres.

Ces expériences itératives des discriminations dans le travail et l'emploi ne font généralement pas sens à elles seules. Elles sont le plus souvent éclairées par des discussions avec des proches, minoritaires ou majoritaires " initiés ${ }^{10}$ ". De plus, l'évolution générale des politiques publiques en France, avec une relative montée en puissance de la thématique des discriminations ethnistes et racistes à partir de la fin des années 1990, rompt avec le déni antérieur et permet de replacer leurs questionnements individuels dans un cadre plus général.

Ce cheminement politique vers un décryptage du racisme, offre une grille permettant d'analyser de nombreux éléments de la vie quotidienne avec un regard critique et argumenté. Il permet de faire le lien entre des expériences individuelles et une vision d'ensemble du social. Il permet aussi de porter un regard rétrospectif sur des expériences passées, à l'école par exemple.

En abordant les entretiens biographiques par leur enfance, les interviewées déclarent, généralement, ne pas avoir rencontré de problème de racisme ou de discrimination à l'école et elles passent rapidement aux études supérieures. Puis, elles en arrivent aux expériences qui fondent leur prise de conscience du racisme et, en conséquence, à l'attention qu'elles portent à la scolarité de leurs enfants pour les protéger. Et du coup, elles reviennent sur leur propre histoire pour faire émerger de nouveaux souvenirs qu'elles soumettent à une analyse critique.

Il ne m'est pas arrivé de grosses mésaventures, il n'y a que des petits malaises. À certains moments on se dit "hum, est-ce que ce n'est pas parce que je suis noire? " Mais c'est tout, c'est un doute. Et puis, une fois qu'on a pris conscience de ça... Moi, là où j'ai quand même observé pas mal, c'est avec des amis [blancs] de mon mari [blanc], que je pensais être des amis proches. Une fois que le doute s'est insinué, on observe leur comportement et on se dit : effectivement ils me voient réellement différente.

Le processus de conscientisation peut aussi amener les interviewées à interroger certaines situations rencontrées dans la sphère privée, notamment ce qui relève des relations affectives ou amicales qui sont si difficilement accessibles à l'observation sociologique. Mes interlocutrices ont mis à profit leurs capacités d'analyse pour relever des comportements typiques qui les maintiennent à l'extérieur du groupe majoritaire et qui préservent

10 Erwing Goffman (1993 [1963]) les définit comme des « compatissants prêts à se mettre à (la) place (des stigmatisés) et à partager avec eux le sentiment qu'ils sont des êtres humains et "essentiellement" normaux, malgré les apparences et quoiqu'ils doutent d'eux-mêmes ». Goffman distingue deux grands modes d'entrée dans la catégorie des compatissants : l'une passe par l'expérience personnelle du stigmate et de la position minoritaire (ce peuvent être, par exemple, les femmes qui projettent leur expérience personnelle sur des individus ou des groupes ethnicisés ou racisés) ; l'autre résulte de leur intimité avec un (ou des) stigmatisé(s), « relation telle que, sous certains rapports, la société en vient à les traiter tous les deux comme s'ils n'en étaient qu'un » (c'est notamment le cas des conjoints ou parents de minoritaires). 
les avantages du groupe dominant. Cet entretien des frontières passe par quelques grands mécanismes récurrents - comme la limitation de l'accès au capital culturel, le contrôle des réseaux sociaux et de l'information, le refus de coopérer, le cantonnement à des tâches concernant d'autres minoritaires, le surcroît d'exigences, le refus de fréquentation ou le contrôle de la circulation dans l'espace national (Poiret, 2010a) - dont la compréhension permet d'affiner l'analyse des processus discriminatoires et ségrégatifs.

Ces observations ont une portée qui va bien au-delà de la seule catégorie « Noire». Elles montrent le rapport entre la construction de la frontière ethnique ou « raciale » et celle de la distance sociale, dès lors que des situations de cohabitation (dans l'école, le travail, l'habitat) sont envisagées. Elles font apparaître une graduation pouvant aller d'une mise à distance symbolique jusqu'à la revendication d'une extériorité totale.

Enfin, la conscientisation peut déboucher sur une réappropriation de la catégorie assignée et sur un éventuel retournement du stigmate dont l'histoire montre qu'ils constituent très souvent une étape nécessaire pour ouvrir la voie à des formes de mobilisation visant à remettre en question un ordre social perçu dans sa dimension racisée. Elle alimente alors le «paradoxe minoritaire », évoqué par Didier et Éric Fassin (2006 : 253), qui fait que le minoritaire est contraint de reprendre à son compte la catégorie qui est au cœur du processus qui le minorise pour tenter de contester celui-ci :

Mon mari est blanc et, vraiment en dehors de ces dernières années, je n'avais presque jamais regardé qu'il était blanc. Presque (bis) [rires] Bon, maintenant je me sens noire et c'est nouveau que je me sente noire. Avant je me sentais Léonore, maintenant je me sens Léonore noire. Et c'est quelque chose que j'ai envie de revendiquer. C'est un sentiment nouveau pour moi, de revendiquer ma différence puisque, de toute façon, on me la renvoie cette différence. Donc j'ai envie de la revendiquer et d'en être fière, quelque part.

Mes interlocutrices disposaient de la formation intellectuelle leur permettant d'expliciter leur analyse et d'envisager des moyens normativement admissibles pour tenter de s'opposer à ces rapports de domination. Mais la révolte des banlieues de novembre 2005 peut aussi être comprise comme une modalité d'expression de cet apprentissage politique, à travers des formes moins sophistiquées ${ }^{11}$.

\section{L'adaptation à la situation subordonnée}

Ceci nous amène à une quatrième et dernière grande forme, l'adaptation à la situation subordonnée (Schwalbe et alii, 2000). On peut distinguer sur le plan conceptuel, avec Danielle Juteau (1999), deux dimensions constitutives des frontières ethniques ou « raciales » : une face interne et l'autre externe qui, dans la réalité, sont indissociables. La face externe est celle posée par le regard des dominants sur les dominés et elle exprime le rapport de domination. La face interne est établie par le regard que portent les dominés sur eux-mêmes, souvent en reprenant les caractéristiques assignées pour tenter de les valoriser.

11 Un programme de recherche est en cours qui visera, notamment, à confronter ces résultats avec ceux obtenus en faisant varier le niveau de diplôme, l'âge et le sexe des personnes enquêtées. 
Parce qu'elles savent qu'elles sont assignées à une situation subordonnée, les personnes minorisées développent des stratégies pour entretenir la face interne de la frontière « raciale » et pour se ménager une situation et une image de soi acceptables. La mise en œuvre de ces réponses stratégiques suppose, avant tout, d'apprendre à contrôler ses affects face à des processus infériorisants ressentis comme des violences.

Toutes savent que lorsqu'elles sont placées dans des situations inacceptables, dans un magasin ou lors d'un entretien d'embauche par exemple, elles doivent prendre garde à mesurer leur protestation. Faute de quoi ce sont elles qui se retrouvent sur la sellette :

Vous devenez le point de mire, donc à chaque fois c'est l'Arabe ou le Noir qui hurle dans un magasin. (...) En fait, après, c'est moi qui me trouve en situation de gêne, parce que les gens vous regardent, parce que les vendeurs passent à autre chose et c'est vous qui avez l'air vraiment stupide de perturber l'atmosphère de la boulangerie ou de l'épicerie.

Car le procès en racisation n'est pas seulement une affaire d'interaction entre deux ou trois individus. Il se déroule sous le regard des membres du groupe majoritaire qui, bien souvent, ne remarquent pas les comportements discriminatoires. Ou qui en sont simplement gênés voire indifférents quand ils ne les trouvent pas normaux. Cette tolérance ou cette approbation tacite constitue d'ailleurs une des principales ressources à la disposition des membres du groupe dominant dans l'entretien de la face externe de la frontière (Essed, 1995 [1991]).

Contrôler ses affects, prendre sur soi, peut signifier tout simplement apprendre à se taire, comme Léonore qui finit par renoncer à discuter des causes de son incapacité à trouver du travail avec son mari et ses amis («blancs ») :

- Les Français n'admettent pas [qu'il y ait des discriminations]. Moi quand j'essaie de discuter de ça avec les copains de mon mari, qu'est-ce que ça les met mal à l'aise.

Question : Et alors, comment ils réagissent? - Ils se ferment, donc au bout d'un moment je me retrouve toute seule à m'enflammer dans des grands discours (...). Ils ne parlent pas, ils se ferment, ils ne répondent pas.

Question : Ils ne vous disent pas que vous exagérez? - Non, ils n'oseraient pas me le dire. C'est ça le drame. Je pense que s'ils étaient à l'aise face à moi, ils me diraient : "Oh mais tu te fais des films, arrêtes... tais-toi! » [rires] Mais non, ils ne disent rien, ils m'écoutent silencieusement, pieusement et donc, au bout d'un moment, j'ai l'impression d'avoir monopolisé la parole et puis d'être vraiment en train de dire des grosses bêtises [rires].

Le refus systématique d'entendre la parole des minoritaires, en leur opposant ici le silence, ailleurs des accusations d'exagération, de paranoïa ou de manque d'humour, est une méthode pédagogique particulièrement efficace pour leur enseigner à se taire. Le rejet de la plainte de la victime, la dénégation de sa souffrance représentent un enjeu non seulement éthique mais aussi social important en ce qu'ils expriment un refus de toute contestation des rapports de domination établis entre racisants et racisés. Mais, en déniant 
aux victimes la possibilité d'exprimer leur souffrance ou leur protestation, il ne s'agit pas seulement de refuser toute contestation des rapports interethniques, il s'agit aussi de redoubler le rapport de domination. La négation de la parole des victimes constitue un processus d'apprentissage. À défaut de s'habituer à la violence raciste, le minoritaire doit apprendre à « faire avec ». C'est ainsi que la surdité du discriminateur finit par rendre le discriminé aphone (Poiret et Vourc'h, 1998).

La gestion de ses affects pour trouver une réponse adaptée est d'autant plus importante que la voie est étroite pour marquer son refus du stigmate sans être discréditée. Mais, au-delà de ces ajustements comportementaux dans les situations d'interaction, l'apprentissage de la racisation passe par des choix tactiques pour répondre au racisme quotidien.

Ces réponses peuvent consister en des formes défensives de renoncement volontaire, sous la contrainte, à certaines ressources ou à certaines perspectives pour pouvoir survivre au sein des institutions majoritaires. Car apprendre à être " Noire ", c'est aussi apprendre la règle de ce que Merton (1997 [1957] : 146) appelait l'alchimie des mœurs, une alchimie sociale qui transmute les qualités des dominants en défaut chez les dominés ${ }^{12}$. Ainsi l'ambition est-elle une vertu majoritaire et un vice minoritaire. Il s'agit alors d'y répondre par des formes d'auto-dévalorisation, comme celle qui consiste à ne pas mentionner ses diplômes les plus élevés dans son curriculum vitae, par exemple.

Dans d'autres cas, ces réponses peuvent amener les personnes racisées à se mettre en retrait de certains réseaux, de certains marchés (y compris du marché matrimonial) ou de certaines institutions majoritaires pour se préserver d'un surcroît d'humiliation qu'elles pourraient y subir. Elles procèdent alors à une auto-exclusion pouvant les conduire à se replier sur une niche ethnique, comme les commerces spécialisés garantissant un certain entre-soi, ou encore par l'amorce d'une carrière déviante, par exemple.

Il faut souligner au passage que ces processus de renoncement ou de mise en retrait ne se situent pas seulement dans l'ordre de la domination symbolique. Ils sont aussi dans l'ordre de l'exploitation : en alimentant des phénomènes de plafond de verre ou d'ethnostratification du travail qui imposent notamment des formes de déqualification et de précarité ; mais aussi en offrant la possibilité de formes de surexploitation qui vont au-delà de la sphère du travail pour s'étendre à l'utilisation intéressée des relations « amicales » ou au domaine de la consommation. C'est le cas, par exemple, du marché du logement où les discriminations garantissent un double avantage aux majoritaires. En tant que locataires, ils bénéficient d'une concurrence moindre sur un marché extrêmement tendu. En tant que bailleurs, la constitution d'un double marché du logement ${ }^{13}$ permet de survaloriser des fractions du parc de logement en recourant à une clientèle captive, quand il ne s'agit pas, purement et simplement, de valoriser des biens dont l'état, voire le statut foncier, ne devrait pas permettre leur mise sur le marché locatif (Poiret, 1996).

12 « Commençons par une formule d'alchimie dont la simplicité est attrayante : la même conduite doit s'évaluer différemment selon les personnes. Par exemple, l'alchimiste expert verra immédiatement que le mot "ferme" se conjugue proprement comme suit : Je suis ferme. Tu es entêté. Il a une tête de cochon... ».

13 Par référence au double marché du travail (Piore, 1979). 
Les réponses à la racisation peuvent aussi relever de tactiques offensives visant à contester la place assignée. Elles ouvrent donc la perspective d'éventuels changements sociaux. Dans notre échantillon, elles s'appuient alors le plus souvent sur une éthique volontariste de l'excellence et du refus de toute posture victimaire dans un jeu de distinction individuelle. Et de la volonté, il en faut pour maîtriser l'image que l'on donne de soi en toutes circonstances et pour toujours apparaître comme une excellente professionnelle « parce que sinon, on va dire, elle est noire, c'est normal ».

Parmi ces tactiques offensives, certaines peuvent viser à contester individuellement la pertinence de la catégorie « Noire» ou de son contenu, pour soi-même mais aussi pour tous ceux qui sont ainsi désignés comme le raconte l'une d'entre elles :

Des PDG, des Directeurs généraux, des Directeurs administratifs, des Directeurs qualité. Quand on allait déjeuner dans des grands restaurants, il fallait que je sois à la hauteur aussi, parce que je représentais la société mais j'avais conscience de représenter aussi la diaspora. Donc, c'est là que j'ai vu que c'était important de faire passer un certain message, de dire : oui, je suis noire, antillaise, africaine. Voilà, on a une représentante, on sait que les Afro-antillais c'est cette

personne-là.

\section{CONCLUSION : ETHNICISATION, RACISATION ET ACTIONS COLLECTIVES}

Les réponses offensives à la racisation peuvent aussi déboucher sur des actions collectives. Cependant, malgré les formes d'identification comme « Noires » qui ressortent des entretiens, force est de constater que, jusqu'à présent, nous n'assistons pas en France à des mobilisations d'ampleur autour de cette catégorie. Les stratégies de distinction, notamment de la part des originaires d'Outre-Mer qui refusent massivement d'endosser la racialisation, restent dominantes pour le moment.

Un élément d'explication revient fréquemment dans les entretiens : il s'agit « d'éviter le communautarisme ». Cette thématique recouvre en fait au moins deux préoccupations distinctes. Éviter le communautarisme peut signifier tout faire pour éviter la ségrégation imposée qui condamne à un entre-soi minoritaire synonyme de privation de ressources. Mais éviter le communautarisme peut aussi vouloir dire éviter d'être soupçonné de communautarisme ; éviter de laisser croire qu'on offre ou qu'on bénéficie de l'appui de membres de son groupe d'appartenance. Car apprendre à être « noire » signifie aussi apprendre que l'on ne peut pas bénéficier à des fins professionnelles, des réseaux familiaux ou amicaux qu'utilisent tout « naturellement» les majoritaires pour trouver un emploi, dès lors qu'il s'agit d'accéder à des fonctions de responsabilités.

C'est ce que découvre Sylviane, diplômée d'une grande école de commerce, lorsqu'elle cherche à se replacer dans le secteur bancaire après avoir connu quelques déboires dans un grand groupe pétrolier. Elle fait intervenir un de ses amis, un cadre de haut niveau centrafricain, à l'appui de sa candidature dans une filiale d'un grand groupe bancaire. Celui-ci, ayant travaillé dans cette entreprise, prend contact avec un de ses 
homologues, « blanc », avec lequel il entretient de longue date des relations conviviales. Et il s'entend répondre : «Alors, malgré le fait que tu es parti, tu veux quand même placer ta tribu? » Ce qui clôt la discussion sur le sujet.

L'accusation de « communautarisme » est l'un des moyens les plus efficaces pour légitimer la stigmatisation d'un groupe racisé dans le contexte de l'idéologie républicaine française (Dhume, 2010). Le battage politique et médiatique autour du thème de la concurrence des victimes mettant en scène des affrontements entre les communautés juive, noire et arabe l'a montré. Ainsi, en mars 2005, la publication par des intellectuels regroupés autour d'Alain Finkielkraut d'un appel contre les « ratonnades anti-blancs » suivie d'une série de déclarations qui conféraient à des catégories racisantes un statut de catégorie légitime d'analyse du social ${ }^{14}$, fut-elle précédée, préparée et autorisée par deux mois de polémique intense sur le thème de la « concurrence des victimes » opposant ces supposées communautés. La constitution de réseaux « non communautaires » représente donc pour les interviewées un enjeu essentiel dans l'élaboration de stratégies offensives d'adaptation à la situation minoritaire. D'où l'importance des associations qui ont pour vocation de favoriser l'insertion des jeunes diplômés ethnicisés et racisés, rebaptisés issus de la diversité. Il en va de même des clubs regroupant des élites de la diversité, comme les Club XXIe siècle ou Averroès ou même le CRAN, qui se défendent de tout communautarisme.

Cette préoccupation s'inscrit dans une évolution des référentiels politiques. Le recours de plus en plus ouvert au registre de la " race » dans les discours publics s'est développé dans un contexte de remise en question des discours officiels sur l'" intégration » et d'émergence des thématiques de la lutte contre les discriminations puis de la promotion de la diversité. Ce changement de registre catégoriel soulève l'hypothèse d'un jeu de bascule entre ethnicisation et racisation des populations désignées comme « noires » qui reflète une évolution de leurs modes de traitement.

Dans le cadre des politiques françaises de gestion des populations minoritaires, l'ethnicisation répond à la perception de " différences » construites comme problèmes professionnels, sociaux et/ou politiques - d'abord à une échelle locale. En se référant à de la culture, de l'acquis, donc à quelque chose qui peut être modifié, elle est fonctionnelle dans le cadre des politiques d'intégration caractérisées par des injonctions à s'assimiler. Ainsi, combiné aux expulsions d'irréguliers, le soutien à des associations communautaires africaines ou à des « médiateurs interculturels » visait et vise toujours à trouver des relais pour tenter de mettre « aux normes » les populations autorisées à séjourner sur le territoire national. L'ethnicisation oriente des formes de gestion locale de la distance perçue qui s'expriment clairement dans les politiques de la Ville mais aussi dans le fonctionnement ethno-stratifié des bassins d'emploi.

14 Le Monde, 24 novembre 2005 : La voix «très déviante 》 d'Alain Finkielkraut au quotidien " Haaretz ». Le philosophe français a assuré au journal israélien que la crise des banlieues était une " révolte ethnico-religieuse ». Il y évoque Dieudonné, « le vrai patron de l'antisémitisme ». «Qu'a fait ce pays aux Africains? Que du bien. À mon père, il a fait subir cinq ans d'enfer. Pourtant, je n'ai jamais été éduqué dans la haine. Or celle des Noirs [contre la France] est pire encore que celle des Arabes ». 
La racisation recrée de la distance avec alter, lorsque les « différences » tendent à disparaître, lorsque les minoritaires ne restent plus « à leur place » et tentent d'entrer en concurrence avec les majoritaires. Parce qu'elle est fondée en nature, la racisation n'appelle pas l'assimilation mais la contention et le confinement. Elle est fonctionnelle dans le cadre des politiques sécuritaires, en désignant les nouvelles classes dangereuses cibles de l'action policière et judiciaire et, plus généralement, de la surveillance des multiples « garde-frontières ethniques »- professionnels du contrôle social (policiers, vigiles, contrôleurs de bus, concierges, " grands-frères ", etc.), semi-professionnels (commerçants, etc.) ou simples quidams - qui contrôlent la circulation à l'intérieur de l'espace national, en développant, au gré des situations, des traitements différenciateurs. Mais la racisation est aussi fonctionnelle dans le cadre des politiques de la diversité qui mettent en scène, à l'échelle nationale voire internationale, de petites élites issues des groupes minorisés, gages de méritocratie venant en contrepoint du traitement discriminatoire et coercitif appliqué à la masse de celles et ceux qui sont vus comme des « Noir-e-s » en France.

Les différents référentiels qui orientent les politiques - publiques et privées, dans le cadre de la gestion des entreprises - ne se substituent pas les uns aux autres. Insertion, intégration, lutte contre les discriminations, égalité des chances, diversité forment un mille-feuilles dont les différentes strates sont disponibles pour être mobilisées selon les circonstances et les objectifs de leurs promoteurs. De même, l'ethnicisation et la racisation se combinent-elles avec des réaffirmations du principe républicain d'aveuglement volontaire à la couleur, pour constituer des formes de minorisation disponibles, simultanément ou successivement, afin d'orienter et/ou de justifier des formes de traitement spécifiques des individus et des groupes minorisés.

\section{Références bibliographiques}

BANTON Michael (1977) The Idea of Race, London, Tavistock Press, $190 \mathrm{p}$.

COHEN William. B (1981) Français et Africains - Les Noirs dans le regard des Blancs, 1530-1880, Paris, Gallimard, 409 p.

DE RUDDER Véronique (1991) Le racisme dans les relations interethniques, L'Homme et la Société, 102, 04/1991, pp. 93-112.

DE RUDDER Véronique et GUILLON Michelle (Collab.) (1987) Autochtones et immigrés en quartier populaire d'Aligre à Îlot Chalon, Paris, CIEMI-L'Harmattan, $234 \mathrm{p}$.

DE RUDDER Véronique, POIRET Christian et VOURC'H François (2000) L'inégalité raciste. L'universalité républicaine à l'épreuve, Paris, PUF, 213 p.

DEWITTE Philippe (1990) Regards blancs et colères noires, Hommes et Migrations, 1132, 05/1990, pp. 3-14.

DHUME Fabrice (2010) L'émergence d'une figure obsessionnelle : comment le « communautarisme » a envahi les discours médiatico-politiques français, Asylon(s), 8, 07/2010. URL : http:// www.reseau-terra.eu/article945.html

DORLIN Elsa (2006) La Matrice de la race. Généalogie sexuelle et coloniale de la Nation française, Paris, La Découverte, 308 p.

DUBOIS William E. B. (2007 [1903]) Les Âmes du peuple noir, Paris, La Découverte, 339 p. 
ESSED Philomena (1995 [1991]) Understanding Everyday Racism. An interdisciplinary Theory, London, Sage Publications, $321 \mathrm{p}$.

FANON Frantz (1952) Peaux noires, masques blancs, Paris, Le Seuil, 237 p.

FASSIN Didier et FASSIN Éric (Dir.) (2006) De la question sociale à la question « raciale »? Représenter la société française, Paris, La Découverte, 264 p.

GOFFMAN Erwing (1975 [1963]) Stigmate. Les usages sociaux des handicaps, Paris, Minuit, $176 \mathrm{p}$.

GUILLAUMIN Colette (1992) Une société en ordre. De quelques unes des formes de l'idéologie raciste, Sociologie et sociétés, XXIV (2), pp. 13-23.

GUILLAUMIN Colette (1994) Un bien vieux néoracisme, Vocabulaire Historique et Critique des Relations Interethniques, 2, Paris L'Harmattan, pp. 5-8

GUILLAUMIN Colette (2002 [1972]) L'idéologie raciste : genèse et langage actuel, Paris, Gallimard, 382 p. (Coll. Folio essais).

GUIRAUDON Virginie (2004) Construire une politique européenne de lutte contre les discriminations : l'histoire de la directive « race », Sociétés contemporaines, 53, pp. 11-32.

HILL COLLINS Patricia (1990) Black Feminist Thought: Knowledge, Consciousness, and the Politics of Empowerment, New York, Routledge, 384 p.

HOOKS bell (1995) Killing rage: ending racism, New York, Henry Holt and, 288 p.

JUTEAU Danielle (1999) De la fragmentation à l'unité. Vers l'articulation des rapports sociaux, in L'ethnicité et ses frontières, Presses de l'Université de Montréal, pp. 103-129

LA PIERE Richard T (1934) Attitude vs Action, Social Forces, 13 (1), pp. 230-237.

MERTON Robert K (1997 [1957]) Eléments de théorie et de méthode sociologique [Traduction et adaptation françaises par H. Mendras], Paris, Armand Colin, 384 p.

MILES M. et HUBERMAN A.-M. (1991) Analyses de données qualitatives : recueil de nouvelles méthodes, Bruxelles, De Boeck, 478 p.

MILES Robert (1989) Racism, London, Routledge, 158 p.

PIORE Michael J (1979) Birds of Passage: Migrant Labor and Industrial Societies. Cambridge, Cambridge University Press, $229 \mathrm{p}$.

POIRET Christian (2010a) Pour une approche processuelle des discriminations : entendre la parole minoritaire, Regard Sociologique, 39, pp. 5-20.

POIRET Christian (2010b) Le retour de la catégorie " Noirs » dans l'espace public français, Migrations Société, 131, pp. 69-85.

POIRET Christian (1996) Familles africaines en France. Ethnicisation, ségrégation, communalisation. Paris, CIEMI-L'Harmattan, $428 \mathrm{p}$.

POIRET Christian et VOURC'H François (1998) Repérer les discriminations ethniques et « raciales » dans le domaine du travail et de l'emploi, Études et Recherches, 165, Montreuil, ISERES, $90 \mathrm{p}$.

SMITH Dorothy (1974) Women's perspective as a radical critique of sociology, Sociological Inquiry, 44, pp. 7-13.

SCHWALBE Michael, GODWIN Sandra, HOLDEN Daphne, SCHROCK Douglas, THOMPSON Shealy and WOLKOMIR Michele (2000) Generic Processes in the Reproduction of Inequality: an Interactionist Analysis, Social Forces, 79 (2), pp. 419-452.

WACQUANT Loïc (1997) For an analytic of racial domination, Political Power and Social Theory, 11, pp. 221-234.

WEST Candace and FENSTERMAKER Sarah (1995) Doing Difference, Gender \& Society, 9, pp. 8-37. 


\title{
Les processus d'ethnicisation et de raci(ali)sation dans la France contemporaine : Africains, Ultramarins et « Noirs »

\author{
Christian Poiret
}

\begin{abstract}
Si les « races » humaines n'existent pas, des groupes racisés existent, constitués d'individus qui tendent à être traités comme l'incarnation de leur groupe d'appartenance et doivent donc apprendre à « faire avec ». Mais plutôt que de considérer ces groupes comme un objet scientifique, cet article leur substitue un objet processuel et contextualisé. La première partie de cet article précise donc les modalités théoriques de construction de cet objet avant de le replacer dans le contexte français contemporain, marqué par le retour de la catégorie « Noirs » dans l'espace public. La deuxième partie présente une approche compréhensive du processus subjectif de racisation par lequel on devient « Noir », basée sur l'analyse de la parole de personnes pouvant être ainsi catégorisées. Ce processus est découpé en quatre grandes formes, liées mais analytiquement distinctes, faisant chacune l'objet d'un développement : la racialisation, l'altérisation, la conscientisation et l'adaptation à la situation subordonnée. Enfin, la troisième partie conclusive revient sur la dimension collective des processus d'ethnicisation et de racisation et leurs conséquences en termes d'action collective.
\end{abstract}

\section{Processes of Ethnicization and Raci(ali)zation in the Contemporary French Context: Africans, Caribbeans and "Blacks"}

\section{Christian Poiret}

If human "races" do not exist, "racized" groups exist that are constituted by individuals who tend to be treated as the embodiment of their group of belonging and thus have to learn to cope with it. Yet rather than considering such groups as scientific object, this article analyzes them as a processual and contextual object. Therefore, the first part of the article clarifies the theoretical construction of such an object and then replaces it in the contemporary French context; this context is marked by the rise of the category "Black" in public space. The second part presents a comprehensive approach of the subjective process of "racization" through which one becomes "Black", based on a discursive analysis among individuals who may be categorized as such. This process is grounded on four main forms that are related but analytically distinct, each of which will be subject to analysis: racialization, otherization, awareness and adaptation to subordination. Finally, the concluding part gets back to the collective dimension of ethnicization and "racization" processes and their implications for collective action. 


\section{Los procesos de etnicización y racialización en la Francia contemporánea: Africanos, Caribeños y «Negros»}

\section{Christian Poiret}

Si bien las «razas» humanas no existen, sí existen grupos «racizados», constituidos por individuos que tienden a ser tratados como la encarnación de su grupo de pertenencia y deben aprender a vivir con estas «características». Este artículo no considera estos grupos como un objeto científico, sino que prefiere trabajar con un objeto analítico procesual y contextualizado. La primera parte de este artículo precisa las modalidades teóricas de construcción de este objeto, y lo sitúa en el contexto francés contemporáneo marcado por el regreso de la categoría «negro/as» en el discurso público. La segunda parte presenta un enfoque comprensivo del proceso subjetivo de racización por el cual uno/ una se convierte en "negro/negra», basado en las voces de las personas que así se pueden categorizar. Este proceso recubre cuatro grandes formas, vinculadas pero analíticamente distintas: la racialización, la alterización, la concientización y la adaptación a la situación subordinada. La tercera parte concluye sobre la dimensión colectiva de los procesos de etnicización y racización y sus consecuencias en términos de acción colectiva. 